\title{
The effects of individual, family and environmental factors on physical activity levels in children: a cross-sectional study
}

\author{
Sharon L Cadogan ${ }^{*}$, Eimear Keane and Patricia M Kearney
}

\begin{abstract}
Background: Physical activity plays an important role in optimising physical and mental health during childhood, adolescence, and throughout adult life. This study aims to identify individual, family and environmental factors that determine physical activity levels in a population sample of children in Ireland.

Methods: Cross-sectional analysis of the first wave (2008) of the nationally representative Growing Up in Ireland study. A two-stage clustered sampling method was used where national schools served as the primary sampling unit (response rate: $82 \%$ ) and age eligible children from participating schools were the secondary units (response rate: $57 \%$ ). Parent reported child physical activity levels and potential covariates (parent and child reported) include favourite hobby, total screen time, sports participation and child body mass index (measured by trained researcher). Univariate and multivariate multinomial logistic regression (forward block entry) examined the association between individual, family and environmental level factors and physical activity levels.
\end{abstract}

Results: The children $(N=8,568)$ were classified as achieving low $(25 \%)$, moderate $(20 \%)$ or high $(55 \%)$ physical activity levels. In the fully adjusted model, male gender (OR 1.64 [95\% Cl: 1.34-2.01]), having an active favourite hobby (OR 1.65 [95\% Cl: 1.31-2.08]) and membership of sports or fitness team (OR 1.90 [95\% Cl: 1.48-2.45]) were significantly associated with being in the high physical activity group. Exceeding two hours total screen time (OR 0.66 [95\% Cl: 0.52-0.85]), being overweight (OR 0.41 [95\%Cl: 0.27-0.61]; or obese (OR 0.68 [95\%Cl: 0.54-0.86]) were significantly associated with decreased odds of being in the high physical activity group.

Conclusions: Individual level factors appear to predict PA levels when considered in the multiple domains. Future research should aim to use more robust objective measures to explore the usefulness of the interconnect that exists across these domains. In particular how the family and environmental settings could be useful facilitators for consistent individual level factors such as sports participation.

Keywords: Physical activity levels, Active, Children, Determinants, Predictors, Individual, Family, Environmental, School

\section{Background}

Physical activity (PA) plays a fundamental role in maintaining and improving physical and mental health, both during childhood and in later years [1,2]. Participating in high levels of PA during childhood produces immediate and, long-term health benefits in adulthood [3,4]. Despite the known health benefits, PA levels decline across the lifespan, particularly during adolescence [3,5-7]. Identified as

\footnotetext{
*Correspondence: scadogan@ucc.ie

Department of Epidemiology and Public Health, University College Cork, Fourth Floor, Western Gateway Building, Western Road, Cork, Ireland
}

the fourth leading risk factor for global mortality [8], physical inactivity is a major public health concern worldwide, associated with an estimated one million deaths annually in the World Health Organisation (WHO) European region alone [9].

WHO international guidelines recommend that children participate in at least 60 minutes of moderate-to-vigorous physical activity (MVPA) daily [10,11]. Worldwide, research has indicated that children are not achieving these guidelines, with estimates of activity levels varying both between and within countries [12-16]. For example, 42\% 
of children aged six to 11 years in the United States [16] participate in 60 minutes of MVPA daily. Similarly, in the United Kingdom (UK), objectively measured PA measurements indicate that just $51 \%$ of four to 10 year olds $(33 \%$ of four to 15 year olds) meet the recommended guidelines [12]. In comparison, $19 \%$ of primary school children and $14 \%$ of 10 to 18 year olds in Ireland meet the recommendations [14].

Achieving the recommended levels of PA per day is essential for the prevention and treatment of many health problems such as obesity. In particular, with evidence of tracking PA from childhood through adolescence and into adulthood [3], developing an active lifestyle from a young age may also produce long term benefits. However, to design effective strategies for increasing children's PA levels, effects on, and determinants of, activity levels need to be well understood.

In order to structure relevant determinants, the conceptual framework for this research adopted Bronfenbrenner's ecological model of child development and well-being $[17,18]$. This model proposes that a child's development is affected by multiple levels of influencers including the micro-system which includes direct influencers such as family, school and neighbourhood [18]. Bronfenbrenner's model advocates the need to address factors at multiple levels in order to understand and change PA behaviours. Multilevel approaches derived from such ecological models have been recommended to examine PA determinants [19].

Existing evidence on correlates of PA in children have been reviewed extensively in the literature $[7,20]$. However, despite the awareness of multi-level associations, many of these factors have been investigated individually. Further, in 2009 , the top five future research priorities for understanding and eliminating disparities in obesity, diet, and PA were published following a meeting of experts in the US [21]. One key recommendation for PA research was to "define individual and environmental factors using mixed methods and other new models to study both simultaneously" [21]. This research uses nationally representative data to examine the multi-level predictive capability of these correlates, specifically; the individual, family, and environmental level factors of PA among nine year olds in Ireland. The first aim of this study is to identify the distribution of individual, family and environmental factors by PA levels. A further novel objective is to model the multi-level effects of these factors on the PA levels of children at age nine.

\section{Methods}

\section{Study design and sample}

The sample comprised of 8,568 nine-year-old schoolchildren participating in the first wave (2008) of Growing Up in Ireland (GUI) study [22]. GUI is a nationally representative cohort of nine year old children living in the Republicof Ireland. The data (in the form of an Anonymised
Microdata File, AMF) are archived in the Irish Social Science Data Archive (ISSDA) and are available to researchers on request.

Eligibility criteria included children who were born between 1st November 1997 and 31st October 1998. The sample was selected using a two-stage clustered sampling method within the Irish primary school system (all mainstream, special and private schools), whereby the school was the primary sampling unit and the age eligible children attending the school were the secondary units $[23,24]$. In the first stage, 1,105 schools from the national total of 3,200 were randomly selected using probability proportionate to size sampling, followed by recruitment of a random sample of eligible children within each school (stage two). At the school level, a response rate of $82.3 \%$ (910 schools) was achieved, while at the level of the household (i.e. eligible child) $57 \%$ of children and their parent/guardians participated in the study. Fieldwork for the school-based component was carried out between March-November 2007, while fieldwork for the home-based phase of data collection ran from July 2007-July 2008. The data were weighted prior to analysis to account for the complex sampling design, which involves the structural adjustment of the sample to the population using Census of Population statistics while maintaining the case base of 8,568 children. More detailed information on the sampling, data collection process and derivation of weights is available elsewhere [24].

Ethical approval was granted by the Health Research Board's Research Ethics Committee based in Dublin, Ireland. Written informed consent was also obtained from a parent or guardian and the study child prior to commencement of the data collection process [24].

\section{Data collection procedures}

Trained social interviewers conducted interviews with the study child and their primary caregiver (and second parent/guardian where applicable) within the home. Parents nominated a primary caregiver (the parent who spent most time with the study child) who was the primary respondent. In $98 \%$ of cases, this was the study child's biological mother. The main interviews were completed on a Computer Assisted Personal Interview (CAPI) basis. There was also a self-complete paper-based supplement for all respondents, which included some potentially sensitive questions such as issues about the marital relationship, marital conflict, experience of depression, and use of drugs [24]. Sources and validity of each of the questions used for this research is contained elsewhere [24]. Anthropometric measurements for the primary and secondary caregiver as well as the study child were also taken during the household interview using standard procedures [24]. 


\section{Dependent variable}

Child PA levels were calculated separately using data reported by the study child's primary caregiver (mother for $98 \%$ of children). The PA questions included in the primary caregivers questionnaire were adapted from the Leisure Time Exercise Questionnaire [25]. The caregiver reported the number of days out of the previous 14 that the child had engaged in 'hard' exercise for at least 20 minutes. Hard exercise was defined as exercise that resulted in heavy breathing and a fast heart beat [23]. This self-report measure has been shown to demonstrate concurrent validity with measures of maximum oxygen intake (VO2 max) and muscular endurance [26], as well as acceptable test-retest reliability [27].

Study child's PA was re-coded into a three level variable based on previous research [28]: low "0-4 days", moderate "5-8 days" and high ">9 days" PA groups. Nine or more days out of previous 14 was the highest possible value and corresponds closest to the recommended PA guidelines. This is also consistent with other Irish research using the same wave of the GUI data [29].

\section{Covariates}

\section{Individual level variables}

Five individual level variables were included: the study child's gender, whether the study child was a member of a sports or fitness club (yes/no), total screen time (TST $[<2$ hours TST per day/ $>2$ hours TST per day]), the nature of study child's favourite hobby (active/inactive) and the study child's weight status (normal/overweight/obese). Data for the former three variables was primary caregiver reported. The study child's favourite hobby variable was based on child reported data. Weight status was classified using objectivity measured data.

TST was categorised based on the recommendations of the American Academy of Paediatricians [30]. This variable was created by combining three screen time variables; hours spent watching TV/videos, playing video games and using a computer ( $<1$ hour, 1-3hours, $>3$ hours). This resulted in a seven level response variable, classified as: "adhering to $(<)$ the recommended maximum two hours/ day" or "exceeding the recommended two hours/day". Adhering to the recommended TST was defined as, the study child only exceeding one hour of screen time in one of the screen time variables (giving a potential for maximum two hours TST).

The study child's favourite hobby variable was created using 32 hobbies listed by the child, classified into a two level response "active" or "inactive" (16 hobbies in each group). A hobby was considered active if it required the child having a physically active participatory role and inactive if the child had a permissive role or remained sedentary. Active hobbies included: basketball, football, hockey and gymnastics. Inactive hobbies included: reading, listening to music and watching TV.
Trained interviewers were responsible for height and weight measurements of each study child and each adult respondent. Height data was recorded to the nearest millimetre using a Leicester portable height stick [24]. Weight was recorded using a SECA 761 flat mechanic scales to the nearest 0.5 kilogram [24]. Children's body mass index (BMI) were classified as normal weight, overweight (BMI of 19.46 for boys and 19.45 for girls) or obese (BMI of 23.39 for boys and 23.46 for girls) using age (9.5 years) and gender specific International Obesity Taskforce (IOTF) cut off points [31].

\section{Family level variables}

Six family level variables were included: primary caregiver's education (third level/post-secondary/ higher secondary/lower secondary or less), employment status (in full time work/ not in full time work), parenting style (authoritative/permissive) primary caregiver weight status (normal, overweight or obese), whether the child has siblings (yes/no) and the household structure (two parent/ single parent). These variables were based on primary caregiver reported data with the exception of objectively measured weight status.

The parenting style variable described the practices of the child's primary caregiver. For the purpose of this research, the original GUI responses; authoritarian, authoritative, permissive and uninvolved parenting styles were recoded as "authoritative" or "permissive". The primary caregiver's measured BMI data was classified according to WHO guidelines as normal weight $\left(<25 \mathrm{~kg} / \mathrm{m}^{2}\right)$, overweight $\left(\geq 25\right.$ and $\left.<30 \mathrm{~kg} / \mathrm{m}^{2}\right)$ or obese $\left(\geq 30 \mathrm{~kg} / \mathrm{m}^{2}\right)$ [32].

\section{Environmental level variables}

Five environmental level variables were included: transport to and from school (active/both active and inactive/ inactive), school playground (good or excellent/fair or poor), school sports facilities (good or excellent/fair or poor), after school activities (agree/disagree) and safe play areas in neighbourhood (agree/disagree).

The school transport variable (caregiver reported) was created using questions on how the study child travelled both to and from school (walks, by public transport, school bus/coach, car, cycles or other). Responses were combined and re-coded as "active both ways", "active one way, inactive one way" and "inactive both ways".

The school playground and sports facilities data were obtained from the school principal questionnaire while data on neighbourhood facilities were primary caregiver reported. Responses for school facilities were re-coded as "very good/excellent" or "fair/poor". Responses to both neighbourhood facilities were re-coded as "agree" or "disagree". 


\section{Statistical analysis}

Secondary analysis was performed using stata (version 12, intercooled). P-values less than 0.05 were considered statistically significant. Probability weights were applied to the data using survey data commands to account for the complex survey design.

Missing data levels were very low for the majority of the variables used, and where missing values were identified (e.g. 5.2\% of PCG BMI measurements) it was found not to be missing at random and hence, data could not be imputed. Primary caregiver reported PA data was available for $99.9 \%$ of the study children, giving an effective case base of 8,566 children for analysis.

Descriptive statistics were performed to evaluate the children's PA related characteristics. Unadjusted multinomial logistic regression methods were used to measure the association between independent predictor variables and moderate/high PA levels. Multinomial multivariate logistic regression was conducted to assess their predictive capability (adjusting for all potential confounders) using the forward block entry function: individual, family and environmental blocks. The first block (model one) included the five individual level factors: gender, weight status, TST, favourite hobby and being a member of a sports or fitness team. Block two (model 2) included the six family level factors: primary caregiver's education, primary caregiver's employment status, primary caregiver's weight status, siblings, parenting style and household structure. Block three (model 3) contained the five environmental level factors: transport to and from school, school's playground facilities, school sports facilities, safe neighbourhood to play in and after school activities.

\section{Results}

\section{Overview of children's PA patterns}

Children were categorised into three PA groups: low $(\mathrm{N}=$ $2,135)$, moderate $(\mathrm{N}=1,740)$ and high $(\mathrm{N}=4,691)$. Overall, 26.3\% (95\% CI, 24.9-27.7) had low, 19.3\% (95\% CI, 18.220.5) had moderate and $54.4 \%$ (95\% CI, 52.8-55.9) had high PA levels. Gender differences existed, with $61 \%(\mathrm{~N}=2,609)$ of boys categorised as being highly active (high PA group) compared to $48 \%(\mathrm{~N}=2,082)$ of girls $(\mathrm{p}<0.001)$. PA/obesity related demographics stratified by gender are presented in Table 1 . Over half of the children $(\mathrm{N}=4,730)$ reported taking exercise almost every day (55\% of boys vs. $45 \%$ of girls, $\mathrm{p}<0.001)$, of which $65 \%(\mathrm{~N}=3,123)$ were in the high, $16 \%(\mathrm{~N}=794)$ in the moderate and $19 \%(\mathrm{~N}=813)$ in the low PA groups $(\mathrm{p}<0.001)$. According to child reported data, $25 \%(\mathrm{~N}=2,136)$ of children met the WHO guidelines of participating in 60 minutes of MVPA each day. Boys were more likely to achieve the recommended guideline than girls $(29 \%$ versus $21 \%, \mathrm{p}<0.001)$. Valid height and weight measurements for the study child were also obtained for $94.5 \%(\mathrm{~N}=8,136)$ of the sample. The estimated proportion of children in the normal, overweight, and obese categories was $74.1 \%$ (95\% CI, 72.8-75.3), 19.3\% (95\% CI, 18.2-20.5) and 6.6\% (95\% CI, 5.9-7.4), res pectively.

\section{Univariate logistic regression findings}

Table 2 presents the results of the univariate multinomial logistic regression. All five of the individual level factors were found to be associated with high PA while four were found to be associated with moderate PA levels. Of the family level factors, primary caregiver's education, primary caregiver's employment status, household structure and parenting style were significantly associated with moderate PA levels, while having siblings and primary caregiver's weight status were not. None of the school level factors were associated with either moderate or high PA levels, while, both safe playgrounds and participating in after school activities in the children's neighbourhood were found to be associated with both moderate and high PA.

\section{Multivariate logistic regression findings}

Figure 1 illustrates the findings (final model) of the multivariate multinomial logistic regression analyses.

\section{Model one (individual level factors)}

Of the individual level factors, male gender $(p<0.001)$, having a physically active favourite hobby $(\mathrm{p}<0.001)$ and being a member of a sports or fitness group $(\mathrm{p}<0.001)$ were positively associated with high PA levels (Table 3). Being a member of a sports or fitness team $(\mathrm{p}<0.001)$ was positively associated with moderate PA. Being overweight or obese was negatively associated with both moderate and high PA, while exceeding the recommended maximum TST (two hours) was negatively associated with high PA $(\mathrm{p}<0.001)$. Obese children were $60 \%$ and $42 \%$ less likely to be in the high and moderate PA groups, respectively (OR, 0.40 [95\% CI, 0.31-0.52] p < 0.001; OR: 0.58 [95\% CI: $0.42-0.79] \mathrm{p}<0.001$ ) compared to children of normal weight. Overweight children were 21\% and 23\% less likely to be in the moderate and high PA groups, respectively (OR, 0.79 [95\% CI: 0.65-0.97] p = 0.02; OR: 0.77 [95\% CI, 0.64-0.91] $\mathrm{p}=0.003)$. Children who exceeded two hours TST were $23 \%$ less likely to be in high PA group (OR, 0.71 [95\% CI, 0.59-0.84] $\mathrm{p}<0.001$ ).

\section{Model two (individual and family level factors)}

None of the family level factors were found to be associated with high PA. Primary caregivers having third level education and an authoritative parenting style were both positively associated with moderate PA levels (Table 3). Children who had primary caregivers with a third level degree were 1.74 times more likely to be in the moderate PA group compared to children of parents with a lower secondary education or less (OR 1.74 [95\% CI: 1.18-2.57] 
Table 1 Physical activity/obesity related characteristics of the children by gender and PA levels

\begin{tabular}{|c|c|c|c|c|c|c|c|c|c|c|}
\hline & & \multicolumn{3}{|c|}{ Boys } & & & \multicolumn{3}{|c|}{ Girls } & \\
\hline & & $\begin{array}{r}\text { Low PA } \\
(\mathrm{N}=826)\end{array}$ & $\begin{array}{r}\text { Moderate PA } \\
\qquad(\mathrm{N}=728)\end{array}$ & $\begin{array}{r}\text { High PA } \\
(\mathrm{N}=2,609)\end{array}$ & & & $\begin{array}{r}\text { Low PA } \\
(\mathrm{N}=1,309)\end{array}$ & $\begin{array}{r}\text { Moderate PA } \\
(\mathrm{N}=1,012)\end{array}$ & $\begin{array}{r}\text { High PA } \\
(\mathrm{N}=2,082)\end{array}$ & \\
\hline Individual factors $^{+}$ & Total & N (\%) & $\mathrm{N}(\%)$ & $\mathrm{N}(\%)$ & p-value & Total & $\mathrm{N}(\%)$ & $\mathrm{N}(\%)$ & $\mathrm{N}(\%)$ & $p$-value \\
\hline Child's weight status** & & & & & $<0.001$ & & & & & $<0.001$ \\
\hline Normal & 3,100 & $558(20)$ & $541(17)$ & $2,001(63)$ & & 3,019 & $812(27)$ & $692(22)$ & 1515(51) & \\
\hline Overweight & 661 & $137(21)$ & $115(17)$ & $409(62)$ & & 875 & $296(36)$ & $204(21)$ & $375(43)$ & \\
\hline Obese & 196 & $74(39)$ & $39(18)$ & $83(43)$ & & 284 & $122(48)$ & $65(20)$ & $97(33)$ & \\
\hline Takes exercise & & & & & $<0.001$ & & & & & $<0.001$ \\
\hline Never & 34 & $22(62)$ & $4(10)$ & $8(28)$ & & 44 & $25(69)$ & $7(10)$ & $12(21)$ & \\
\hline 1-2times/week & 673 & $243(38)$ & $144(20)$ & $286(42)$ & & 957 & $446(47)$ & $223(21)$ & $288(32)$ & \\
\hline 3-4times/week & 939 & $209(25)$ & $234(25)$ & $496(51)$ & & 1,136 & $356(33)$ & $329(27)$ & $453(40)$ & \\
\hline Almost every day & 2,486 & $341(16)$ & $344(14)$ & $1801(70)$ & & 2,244 & $472(22)$ & $450(19)$ & $1,322(59)$ & \\
\hline Sports/fitness club & & & & & $<0.001$ & & & & & $<0.001$ \\
\hline Yes & 3,585 & $596(18)$ & $644(18)$ & $2345(64)$ & & 3,137 & $809(26)$ & 768 (24) & $1,560(49)$ & \\
\hline No & 573 & $226(41)$ & $84(14)$ & $263(45)$ & & 1,261 & $496(40)$ & $244(16)$ & $521(44)$ & \\
\hline Playing sport* & & & & & $<0.001$ & & & & & 0.11 \\
\hline Favourite ${ }^{\#}$ & 1,657 & $232(15)$ & $258(16)$ & $1167(69)$ & & 809 & $178(23)$ & $195(24)$ & $436(53)$ & \\
\hline Second favourite & 968 & $155(17)$ & $187(19)$ & $636(64)$ & & 767 & $207(30)$ & $179(22)$ & $381(48)$ & \\
\hline Third favourite & 455 & $97(27)$ & $78(16)$ & $280(57)$ & & 506 & $144(31)$ & $123(22)$ & $239(48)$ & \\
\hline Watching TV* & & & & & 0.38 & & & & & 0.99 \\
\hline Favourite & 169 & $53(29)$ & $33(19)$ & $83(52)$ & & 195 & $73(35)$ & $42(20)$ & $80(45)$ & \\
\hline Second favourite & 491 & $126(28)$ & $88(20)$ & $277(53)$ & & 428 & $144(34)$ & $94(21)$ & $190(45)$ & \\
\hline Third favourite & 669 & $135(23)$ & $128(18)$ & $406(59)$ & & 551 & $187(35)$ & $121(21)$ & $243(44)$ & \\
\hline Playing video games* & & & & & $<0.001$ & & & & & 0.36 \\
\hline Favourite & 211 & $71(37)$ & $42(17)$ & $98(46)$ & & 84 & $34(35)$ & $22(29)$ & $28(36)$ & \\
\hline Second favourite & 318 & $84(30)$ & $64(19)$ & $170(51)$ & & 202 & $61(28)$ & $54(24)$ & $87(48)$ & \\
\hline Third favourite & 392 & $83(21)$ & $70(18)$ & $239(62)$ & & 255 & $86(36)$ & $58(20)$ & $111(43)$ & \\
\hline Watching TV & & & & & $<0.001$ & & & & & $<0.001$ \\
\hline Zero or $<1$ hour & 1,050 & $157(17)$ & $174(15)$ & $719(68)$ & & 1,186 & $274(25)$ & $279(21)$ & $633(54)$ & \\
\hline 1-3hours & 2,723 & $539(21)$ & $475(18)$ & $1,709(62)$ & & 2,819 & $856(30)$ & $656(22)$ & $1,307(47)$ & \\
\hline$>3$ hours & 390 & $130(35)$ & $79(21)$ & $181(44)$ & & 398 & $179(45)$ & $77(19)$ & $142(36)$ & \\
\hline Playing video games & & & & & $<0.001$ & & & & & 0.02 \\
\hline Zero or $<1$ hour & 3,059 & $549(20)$ & $522(17)$ & $1,988(63)$ & & 3,923 & 1,118 (29) & $923(22)$ & $1,882(48)$ & \\
\hline 1-3hours & 1,011 & $245(26)$ & $185(17)$ & $581(57)$ & & 438 & $169(37)$ & $82(16)$ & $187(47)$ & \\
\hline$>3$ hours & 93 & $32(38)$ & $21(21)$ & $40(41)$ & & 39 & $19(46)$ & $7(21)$ & $13(32)$ & \\
\hline On the computer & & & & & $<0.001$ & & & & & 0.34 \\
\hline Zero or $<1$ hour & 3,650 & $669(21)$ & $624(17)$ & $2,337(62)$ & & 3,820 & $1,097(30)$ & $895(22)$ & $1,828(48)$ & \\
\hline 1-3hours & 498 & $143(31)$ & $94(17)$ & $261(52)$ & & 549 & $200(35)$ & $112(44)$ & $237(44)$ & \\
\hline$>3$ hours & 33 & $14(33)$ & $9(31)$ & $10(36)$ & & 32 & $11(31)$ & $5(15)$ & $16(53)$ & \\
\hline Total screen time & & & & & $<0.001$ & & & & & 0.002 \\
\hline$<2$ hours/day & 899 & $128(17)$ & $142(14)$ & $629(69)$ & & 1,082 & $247(25)$ & $258(22)$ & $577(53)$ & \\
\hline$>2$ hours/day & 3,262 & $698(23)$ & 585 (18) & 1,979 (59) & & 3,317 & $1,059(32)$ & $754(22)$ & $1,504(46)$ & \\
\hline
\end{tabular}

Family factors ${ }^{+}$ 
Table 1 Physical activity/obesity related characteristics of the children by gender and PA levels (Continued)

\begin{tabular}{|c|c|c|c|c|c|c|c|c|c|}
\hline Caregiver's weight*** & & & & & 0.74 & & & & 0.13 \\
\hline Normal & 1,925 & $349(21)$ & $340(17)$ & $1,236(62)$ & 1,962 & $552(29)$ & $466(21)$ & $944(50)$ & \\
\hline Overweight & 1,244 & $262(22)$ & $224(18)$ & $758(60)$ & 1,300 & 391 (31) & $282(21)$ & $627(47)$ & \\
\hline Obese & 655 & $149(23)$ & $112(17)$ & $394(60)$ & 1735 & $248(35)$ & $172(23)$ & $315(43)$ & \\
\hline Caregiver's education & & & & & 0.01 & & & & $<0.001$ \\
\hline$</=$ lower second level & 674 & $156(25)$ & $108(15)$ & $410(60)$ & 834 & $281(35)$ & $151(17)$ & $402(48)$ & \\
\hline Higher second level & 1,295 & $287(23)$ & $248(19)$ & $760(58)$ & 1,403 & $428(31)$ & $319(22)$ & $656(47)$ & \\
\hline Post second level & 1,056 & $203(21)$ & $173(16)$ & $680(63)$ & 1,067 & $302(27)$ & $241(23)$ & $524(50)$ & \\
\hline Third level & 1,138 & $180(17)$ & $199(17)$ & 759 (66) & 1,099 & $298(26)$ & $301(27)$ & $500(47)$ & \\
\hline Siblings & & & & & 0.59 & & & & 0.17 \\
\hline Yes & 3,716 & $728(22)$ & $656(18)$ & $2,332(61)$ & 3,977 & $1,166(30)$ & $910(21)$ & $1,901(49)$ & \\
\hline No & 329 & $66(20)$ & $57(16)$ & $206(64)$ & 330 & $101(32)$ & $88(26)$ & $141(42)$ & \\
\hline Household type & & & & & 0.01 & & & & 0.71 \\
\hline Single Parent & 457 & $119(28)$ & $58(14)$ & $280(59)$ & 534 & $165(32)$ & $118(20)$ & $251(48)$ & \\
\hline Two parent & 3,706 & $707(21)$ & $670(18)$ & $2,329(61)$ & 3,869 & $1,144(31)$ & $894(22)$ & $1,831(47)$ & \\
\hline \multicolumn{10}{|l|}{ Environmental factors ${ }^{+}$} \\
\hline Transport to school & & & & & 0.21 & & & & 0.68 \\
\hline Active & 1,047 & $199(23)$ & $167(15)$ & $681(62)$ & 1,085 & $298(30)$ & $261(22)$ & $526(49)$ & \\
\hline Inactive & 3,116 & $627(22)$ & $561(18)$ & $1,928(60)$ & 3,318 & $1,011(31)$ & $751(21)$ & $1,556(47)$ & \\
\hline Transport from school & & & & & 0.24 & & & & 0.48 \\
\hline Active & 1,160 & $223(23)$ & $190(15)$ & $747(63)$ & 1,209 & $339(30)$ & $298(23)$ & $572(48)$ & \\
\hline Inactive & 3,003 & $603(21)$ & $538(18)$ & $1,862(60)$ & 3,189 & $967(31)$ & $713(21)$ & $1,509(48)$ & \\
\hline School playground^ & & & & & 0.19 & & & & 0.79 \\
\hline Fair/poor & 1,660 & $320(22)$ & $316(19)$ & $1,024(59)$ & 1,704 & $501(30)$ & $398(22)$ & $805(48)$ & \\
\hline Good/excellent & 2,361 & $453(22)$ & $389(16)$ & $1,489(62)$ & 2,493 & $751(31)$ & $573(21)$ & $1,169(48)$ & \\
\hline School sports facilities^ & & & & & 0.97 & & & & 0.43 \\
\hline Fair/poor & 1,765 & $343(22)$ & $310(17)$ & $1,112(60)$ & 1,908 & $573(31)$ & $417(20)$ & $918(48)$ & \\
\hline Good/excellent & 2,267 & $460(22)$ & $397(17)$ & $1,410(61)$ & 2,341 & $698(31)$ & $563(22)$ & $1080(47)$ & \\
\hline Safe places to play & & & & & 0.34 & & & & 0.18 \\
\hline Agree & 3,814 & $740(22)$ & $662(17)$ & $2412(61)$ & 4,016 & $1,194(30)$ & $925(22)$ & $1,897(48)$ & \\
\hline Disagree & 344 & $85(26)$ & $64(16)$ & $195(58)$ & 113 & $381(36)$ & $87(19)$ & $181(46)$ & \\
\hline
\end{tabular}

${ }^{+}$All data is primary caregiver reported unless indicated otherwise.

${ }^{*}$ Child-reported variable.

**Weight status defined as BMI classified according to International Obesity Taskforce on Obesity age and gender specific guidelines using objectively measured height and weight data.

***Weight status defined as BMI classified according to World Health Organisation guidelines using objectively measured height and weight data. $\wedge$ School principal reported data.

${ }^{\#}$ Favourite refers to the study child reporting the hobby as being their favourite thing to do.

$\mathrm{p}<0.01)$. Having a primary caregiver who adopts an authoritative parenting style was associated with a $42 \%$ increase in the child's probability of being in the moderate PA group (OR 1.42 [95\% CI: 1.06-1.87] $\mathrm{p}=0.02$ ) compared to having a primary caregiver with a permissive parenting style.

In model two, the strength of the association for three of the significant individual level factors (gender, weight status and being a member of a sports or fitness team) became stronger. In particular, the probability of being in the high PA group was 66\% higher for boys (OR: 1.66 [95\% CI: 1.37-2.01] $\mathrm{p}<0.01$ ).

\section{Model three (fully adjusted model)}

Accounting for both individual and family level factors, active travel to and from school was positively associated with high PA levels. A positive association between living in a neighbourhood with after school activities and moderate PA was also identified. Children who used active mode of travel both to and from school were $34 \%$ more likely to 
Table 2 Independent association of each of the individual, family and environmental level factors and moderate or high PA levels

\begin{tabular}{|c|c|c|c|c|}
\hline \multirow[t]{2}{*}{ Variable } & \multicolumn{2}{|c|}{ Moderate $\mathrm{PA}^{*}(\mathrm{~N}=1,740)$} & \multicolumn{2}{|c|}{ High $P A^{*}(N=4,691)$} \\
\hline & OR $(95 \% \mathrm{Cl})$ & p-value & OR $(95 \% \mathrm{Cl})$ & p-value \\
\hline \multicolumn{5}{|l|}{ Individual factors $^{+}$} \\
\hline \multicolumn{5}{|l|}{ Gender } \\
\hline Boys & $1.13(0.96-1.33)$ & 0.14 & $1.79(1.55-2.07)$ & $<0.001$ \\
\hline Girls & $1 * * *$ & & 1 & \\
\hline \multicolumn{5}{|l|}{ Child's weight status $^{\#}$} \\
\hline Obese & $0.52(0.38-0.71)$ & $<0.001$ & $0.34(0.26-0.44)$ & $<0.001$ \\
\hline Overweight & $0.78(0.64-0.95)$ & 0.01 & $0.71(0.60-0.84)$ & $<0.001$ \\
\hline Normal & 1 & & 1 & \\
\hline \multicolumn{5}{|l|}{ Child's favourite hobby** } \\
\hline Active hobby^ & $1.26(1.08-1.48)$ & 0.01 & $1.81(1.57-2.08)$ & $<0.001$ \\
\hline Inactive hobby & 1 & & 1 & \\
\hline \multicolumn{5}{|l|}{ Sports/fitness club } \\
\hline Yes & $2.49(2.09-2.98)$ & $<0.001$ & $2.41(2.0-2.82)$ & $<0.001$ \\
\hline No & 1 & & 1 & \\
\hline \multicolumn{5}{|l|}{ Total screen time } \\
\hline$<$ Recommended 2 hours & $0.83(0.67-1.01)$ & 0.06 & $0.66(0.56-0.78)$ & $<0.001$ \\
\hline$>$ Recommended 2 hours & 1 & & 1 & \\
\hline \multicolumn{5}{|l|}{ Family factors $^{+}$} \\
\hline \multicolumn{5}{|l|}{ Caregiver's employment } \\
\hline Not in full time & $1.31(1.02-1.69)$ & 0.04 & $1.23(0.99-1.53)$ & 0.06 \\
\hline In full time work & 1 & & 1 & \\
\hline \multicolumn{5}{|l|}{ Caregiver's education } \\
\hline Third level & $1.93(1.51-2.46)$ & $<0.001$ & $1.56(1.28-1.90)$ & $<0.001$ \\
\hline Post-secondary & $1.48(1.17-1.87)$ & $<0.001$ & $1.35(1.13-1.62)$ & 0.001 \\
\hline Higher secondary & $1.45(1.16-1.79)$ & $<0.001$ & $1.10(0.94-1.32)$ & 0.23 \\
\hline$<=$ Lower secondary & 1 & & 1 & \\
\hline \multicolumn{5}{|l|}{ Caregiver's weight status \#\# } \\
\hline Obese & $0.86(0.68-1.09)$ & 0.21 & $0.77(0.64-0.94)$ & 0.01 \\
\hline Overweight & $0.96(0.79-1.17)$ & 0.69 & $0.89(0.76-1.04)$ & 0.13 \\
\hline Normal & 1 & & 1 & \\
\hline \multicolumn{5}{|l|}{ Siblings } \\
\hline Yes & $0.92(0.67-1.25)$ & 0.57 & $1.02(0.78-1.32)$ & 0.90 \\
\hline No & 1 & & 1 & \\
\hline \multicolumn{5}{|l|}{ Household type } \\
\hline Two parent & $1.37(1.07-1.75)$ & 0.01 & $1.19(0.99-1.45)$ & 0.06 \\
\hline Single parent & 1 & & 1 & \\
\hline \multicolumn{5}{|l|}{ Parenting style } \\
\hline Authoritative & $1.26(1.03-1.55)$ & 0.02 & $1.22(1.03-1.44)$ & 0.02 \\
\hline Permissive & 1 & & 1 & \\
\hline
\end{tabular}




\begin{tabular}{|c|c|c|c|c|}
\hline \multicolumn{5}{|c|}{ Environmental factors ${ }^{+}$} \\
\hline \multicolumn{5}{|l|}{ Travel to/from school } \\
\hline Active both ways & $0.94(0.77-1.15)$ & 0.54 & $1.02(0.87-1.20)$ & 0.80 \\
\hline Active one way & $1.12(0.83-1.52)$ & 0.46 & $1.00(0.79-1.28)$ & 0.99 \\
\hline Inactive both ways & 1 & & 1 & \\
\hline \multicolumn{5}{|l|}{ School playground*** } \\
\hline Good/excellent & $0.89(0.74-1.06)$ & 0.18 & $1.00(0.86-1.18)$ & 0.94 \\
\hline Fair/poor & 1 & & 1 & \\
\hline \multicolumn{5}{|c|}{ School sports facilities ${ }^{* * *}$} \\
\hline Good/excellent & $1.08(0.91-1.28)$ & 0.37 & $1.03(0.88-1.20)$ & 0.75 \\
\hline Fair/poor & 1 & & 1 & \\
\hline \multicolumn{5}{|l|}{ Safe places to play } \\
\hline Agree & $1.20(1.02-1.41)$ & 0.03 & $1.20(1.05-1.38)$ & 0.01 \\
\hline Disagree & 1 & & 1 & \\
\hline \multicolumn{5}{|l|}{ After school activities } \\
\hline Yes & $1.29(1.07-1.56)$ & 0.01 & $1.22(1.04-1.42)$ & 0.01 \\
\hline No & 1 & & 1 & \\
\hline
\end{tabular}

${ }^{+}$All data is primary caregiver reported unless indicated otherwise.

* Reference category: low PA.

**Child-reported data.

***School principal reported data.

****1 denotes reference category.

$\wedge$ Active hobby was defined as one in which the study child had a physically active participatory role.

"Weight status defined as BMI classified according to International Obesity Taskforce on Obesity age and gender specific guidelines using objectively measured height and weight data.

\#\#Weight status defined as BMI classified according to World Health Organisation guidelines using objectively measured height and weight data.

be in the high PA group (OR 1.34 [95\% CI: 1.03-1.74] $\mathrm{p}=$ 0.03) compared to children who used an inactive mode of travel both to and from school. Children living in a neighbourhood with after school activities were 39\% more likely to be in the moderate PA group compared to those who lived in neighbourhoods without after school activities (OR 1.39 [95\% CI: 1.05-1.84] $\mathrm{p}=0.02$ ).

The association between the individual level factors and high PA remained statistically significant. Of the family level factors, having a primary caregiver with third level education and authoritative parenting styles remained positively associated with moderate PA levels. None of the family level factors were associated with high PA.

\section{Discussion}

To our knowledge, this is the first study to explore the multi-level effects of individual, family and environmental factors on PA levels of children in Ireland. A key finding of this research is that individual level factors appear to have the strongest association with PA levels in nine year olds. Further, many of these factors are modifiable. Being a member of a sports or fitness club, and, having an active favourite hobby were both positively associated with higher levels of PA. Exceeding two hours of TST and being overweight or obese were negatively correlated with higher PA levels. No significant associations with the family level and just one marginal association among the environmental level factors were identified. However, environmental level factors could provide cost effective settings for implementing PA initiatives such as supporting sports participation.

Consistent with both extensive reviews by Sallis et al. [7] and van der Horst et al. [20], boys were more likely to have high PA levels. Literature suggests that differences in organised sports participation may be responsible for some of gender disparities in PA levels. In this research, over $75 \%$ of the children were members of a sports or fitness group ( $84 \%$ of boys versus $67 \%$ of girls, $\mathrm{p}=0.000$ ). In the fully adjusted model (controlled for gender), this research found children who were members of a sports or fitness group were almost twice as likely to be in the high PA group compared to children who were not. This is consistent with findings of the review by Sallis et al. which concluded that community sports participation [7] was positively associated with higher PA levels. Despite generally higher sports participation among boys, a review of PA correlates among girls aged 

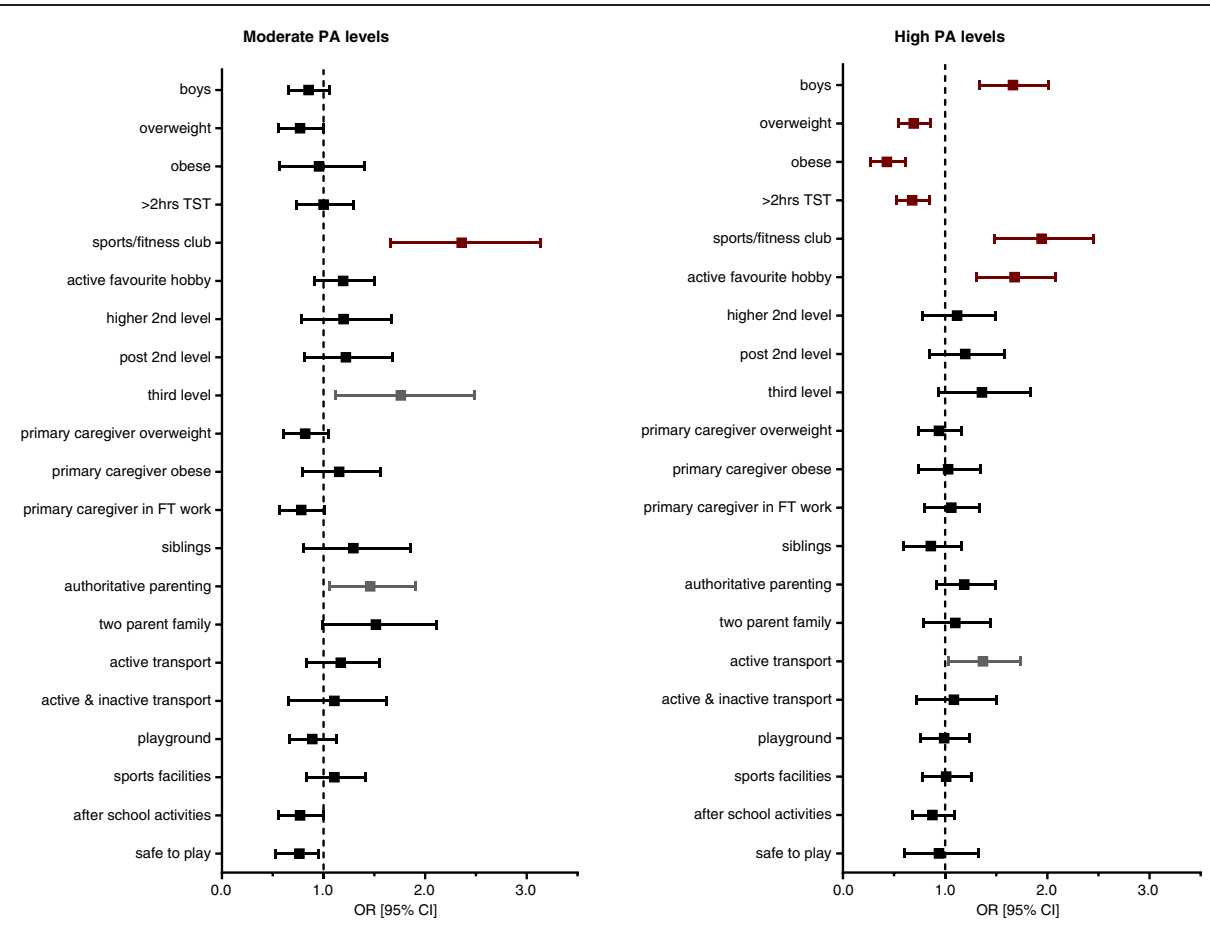

Figure 1 Individual, family and environmental factors associated with moderate and high physical activity.

between 10 and 18 also found that organised sports participation had a consistent positive association with higher PA levels [33]. Moreover, longitudinal studies have reported that participation in organised sports during childhood may be associated with long-term participation in PA in both adolescence and adulthood [3,34]. The promotion of sports and other high intensity activities may therefore provide an opportunity to increase PA among school children.

Many sports and other high intensity activities take place as extra-curricular activities after school hours. The Irish primary school day typically lasts five hours and 40 minutes, commencing at 9 am and finishing at approximately $3 \mathrm{pm}$. While the curriculum recommends one hour of physical education per week, it has been suggested that many schools do not provide this [14]. As a result, children's preferences for extracurricular activities may also play a role in their overall PA levels. This research found that children reporting a preference for an active favourite hobby (including basketball, gymnastics and hockey) were more likely to be in the high PA group compared to children who preferred inactive favourite hobbies such as reading, listening to music, and watching TV. Similarly, in their review of previous research, Sallis et al. [7] concluded that children's preference for physical (rather than sedentary) activity was one of the factors most consistently associated with their participation in such activity.
Another key factor that may be associated with PA levels among nine year olds is sedentary behaviour. The American Academy of Paediatricians recommends that children do not exceed two hours of sedentary screen time per day [30]. Previous Irish research reported that over $99 \%$ of children and youth exceeded the recommended maximum two hours sedentary screen time per day [14]. Conflicting evidence exists for an association between sedentary behaviours (including screen time) and PA levels among children $[7,35]$. This present research found that exceeding these guidelines reduced the likelihood of high PA by $44 \%$. The literature refers to the displacement theory as a possible explanation for an association between exceeding the recommended and lower PA, that is, sedentary behaviours may be replacing active behaviours [36].

PA behaviour and the factors influencing it are very complex. The social-ecological model adopted by this present research is a useful framework due to the complexity of behaviours [18]. Each level of the model layers (individual, family and environmental) is interconnected. Exploring the multiple domains, this present research has considered the broader context when identifying the predictors of PA. While this research did not identify environmental factors as major determinants of PA, more research is needed. In particular, the importance of built environments for 
Table 3 Multivariate multinomial analysis of the individual, family and environmental factors and PA levels

\begin{tabular}{|c|c|c|c|c|c|c|c|c|c|c|c|c|}
\hline & \multicolumn{4}{|c|}{ Model 1 (individual level factors) } & \multicolumn{4}{|c|}{ Model 2 (model 1 + family level factors) } & \multicolumn{4}{|c|}{ Model 3 (model 1 and $2+$ environmental factors) } \\
\hline & \multicolumn{2}{|c|}{ Moderate } & \multicolumn{2}{|c|}{ High } & \multicolumn{2}{|c|}{ Moderate } & \multicolumn{2}{|c|}{ High } & \multicolumn{2}{|c|}{ Moderate } & \multicolumn{2}{|c|}{ High } \\
\hline & OR $(95 \% \mathrm{Cl})$ & $\mathrm{p}$-value & OR (95\%Cl) & $\mathrm{p}$-value & OR $(95 \% \mathrm{Cl})$ & $\mathrm{p}$-value & OR (95\%Cl) & $\mathrm{p}$-value & OR (95\%Cl) & $\mathrm{p}$-value & OR $(95 \% \mathrm{Cl})$ & $\mathrm{p}$-value \\
\hline \multicolumn{13}{|l|}{ Individual factors ${ }^{+}$} \\
\hline \multicolumn{13}{|l|}{ Gender } \\
\hline Boys & $0.96(0.81-1.14)$ & 0.63 & $1.54(1.32-1.80)$ & $<0.001$ & $0.85(0.68-1.08)$ & 0.19 & $1.66(1.37-2.01)$ & $<0.001$ & $0.84(0.66-1.06)$ & 0.15 & $1.64(1.34-2.01)$ & $<0.001$ \\
\hline Girls & $1^{* * *}$ & & 1 & & 1 & & 1 & & 1 & & 1 & \\
\hline \multicolumn{13}{|l|}{ 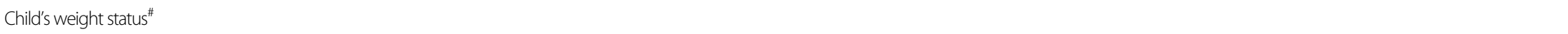 } \\
\hline Obese & $0.58(0.42-0.79)$ & $<0.001$ & $0.40(0.31-0.52)$ & $<0.001$ & $0.86(0.55-1.34)$ & 0.51 & $0.41(0.27-0.61)$ & $<0.001$ & $0.90(0.57-1.40)$ & 0.63 & $0.41(0.27-0.61)$ & $<0.001$ \\
\hline Overweight & $0.79(0.65-0.97)$ & 0.02 & $0.77(0.64-0.91)$ & 0.001 & $0.72(0.55-1.34)$ & 0.02 & $0.68(0.54-0.85)$ & $<0.001$ & $0.75(.56-1.00)$ & 0.05 & $0.68(0.54-0.86)$ & $<0.001$ \\
\hline Normal & 1 & & 1 & & 1 & & 1 & & 1 & & 1 & \\
\hline \multicolumn{13}{|l|}{ Total screen time^ } \\
\hline$<$ Recommended 2 hours & $0.90(0.73-1.11)$ & 0.33 & $0.71(0.59-0.84)$ & $<0.001$ & $0.97(0.73-1.28)$ & 0.82 & $0.67(0.53-0.86)$ & $<0.001$ & $0.97(0.73-1.30)$ & 0.85 & $0.66(0.52-0.85)$ & $<0.001$ \\
\hline$>$ Recommended 2 hours & 1 & & 1 & & 1 & & 1 & & 1 & & 1 & \\
\hline \multicolumn{13}{|l|}{ Favourite hobby* } \\
\hline Active & $1.13(0.95-1.35)$ & 0.27 & $1.65(1.42-1.92)$ & $<0.001$ & $1.21(0.95-1.53)$ & 0.13 & $1.62(1.30-2.03)$ & $<0.001$ & $1.17(0.91-1.50)$ & 0.21 & $1.65(1.31-2.08)$ & $<0.001$ \\
\hline Inactive & 1 & & & & 1 & & 1 & & 1 & & 1 & \\
\hline \multicolumn{13}{|l|}{ Sports/fitness club } \\
\hline Yes & $2.28(1.88-2.77)$ & $<0.001$ & $1.86(1.58-2.20)$ & $<0.001$ & $2.32(1.69-3.18)$ & $<0.001$ & $1.92(1.50-2.46)$ & $<0.001$ & $2.28(1.66-3.14)$ & 0.001 & $1.90(1.48-2.45)$ & $<0.001$ \\
\hline No & 1 & & 1 & & 1 & & & & 1 & & 1 & \\
\hline \multicolumn{13}{|l|}{ Family factors $^{+}$} \\
\hline \multicolumn{13}{|l|}{ Caregiver's education } \\
\hline Third level & & & & & $1.74(1.18-2.57)$ & 0.01 & $1.32(0.96-1.81)$ & 0.09 & $1.67(1.12-2.50)$ & 0.01 & $1.31(0.94-1.83)$ & 0.11 \\
\hline Post-secondary & & & & & $1.21(0.85-1.72)$ & 0.29 & $1.17(0.87-1.57)$ & 0.31 & $1.14(0.78-1.67)$ & 0.46 & $1.16(0.85-1.58)$ & 0.34 \\
\hline Higher secondary & & & & & $1.17(0.81-1.68)$ & 0.41 & $1.09(0.80-1.47)$ & 0.60 & $1.16(0.81-1.68)$ & 0.42 & $1.08(0.79-1.49)$ & 0.62 \\
\hline$<=$ Lower secondary & & & & & 1 & & 1 & & 1 & & 1 & \\
\hline \multicolumn{13}{|l|}{ Caregiver's employment } \\
\hline In full time work & & & & & $0.81(0.61-1.08)$ & 0.15 & $1.05(0.82-1.34)$ & 0.73 & $0.76(0.57-1.01)$ & 0.06 & $1.04(0.72-1.50)$ & 0.79 \\
\hline Not in full time & & & & & 1 & & 1 & & 1 & & 1 & \\
\hline \multicolumn{13}{|l|}{ Caregiver's weight ${ }^{\#}$} \\
\hline Obese & & & & & $1.14(0.82-1.58)$ & 0.43 & $1.02(0.76-1.36)$ & 0.90 & $1.11(0.79-1.56)$ & 0.54 & $1.00(0.74-1.35)$ & 0.99 \\
\hline
\end{tabular}


Table 3 Multivariate multinomial analysis of the individual, family and environmental factors and PA levels (Continued)

\begin{tabular}{|c|c|c|c|c|c|c|c|c|}
\hline Overweight & $0.85(0.65-1.11)$ & 0.23 & $0.94(0.75-1.17)$ & 0.56 & $0.80(0.61-1.05)$ & 0.11 & $0.92(0.74-1.16)$ & 0.48 \\
\hline Normal & 1 & & 1 & & 1 & & 1 & \\
\hline \multicolumn{9}{|l|}{ Siblings } \\
\hline Yes & $1.20(0.80-1.79)$ & 0.37 & $0.86(0.62-1.18)$ & 0.35 & $1.22(0.80-1.86)$ & 0.35 & $0.83(0.59-1.16)$ & 0.28 \\
\hline No & 1 & & 1 & & 1 & & 1 & \\
\hline \multicolumn{9}{|l|}{ Parenting style } \\
\hline Authoritative & $1.41(1.07-1.87)$ & 0.02 & $1.15(0.90-1.47)$ & 0.25 & $1.42(1.06-1.90)$ & 0.02 & $1.16(0.91-1.49)$ & 0.23 \\
\hline Permissive & 1 & & 1 & & 1 & & 1 & \\
\hline \multicolumn{9}{|l|}{ Household type } \\
\hline Two parent & $1.38(0.96-1.98)$ & 0.08 & $1.03(0.77-1.38)$ & 0.82 & $1.45(0.99-2.12)$ & 0.06 & $1.07(0.79-1.44)$ & 0.74 \\
\hline One parent & 1 & & 1 & & 1 & & 1 & \\
\hline \multicolumn{9}{|l|}{ Environmental factors ${ }^{+}$} \\
\hline \multicolumn{9}{|l|}{ Travel to/from school } \\
\hline Active both ways & & & & & $1.13(0.83-1.41)$ & 0.43 & $1.34(1.03-1.74)$ & 0.03 \\
\hline Active one way & & & & & $1.04(0.66-1.62)$ & 0.88 & $1.04(0.72-1.50)$ & 0.85 \\
\hline Inactive both ways & & & & & 1 & & 1 & \\
\hline \multicolumn{9}{|l|}{ School playground** } \\
\hline Good/excellent & & & & & $0.87(0.67-1.62)$ & 0.29 & $0.97(0.76-1.24)$ & 0.82 \\
\hline Fair/poor & & & & & 1 & & 1 & \\
\hline \multicolumn{9}{|c|}{ School sports facilities** } \\
\hline Good/excellent & & & & & $1.08(0.83-1.41)$ & 0.56 & $0.99(0.78-1.26)$ & 0.95 \\
\hline Fair/poor & & & & & 1 & & 1 & \\
\hline \multicolumn{9}{|l|}{ Safe places to play } \\
\hline Agree & & & & & $0.81(0.53-1.24)$ & 0.33 & $1.12-0.75-1.66)$ & 0.58 \\
\hline Disagree & & & & & 1 & & 1 & \\
\hline \multicolumn{9}{|l|}{ After school activities } \\
\hline Yes & & & & & $1.39(1.05-1.84)$ & 0.02 & $1.16(0.92-1.46)$ & 0.22 \\
\hline No & & & & & 1 & & 1 & \\
\hline
\end{tabular}

${ }^{+}$All data is primary caregiver reported unless indicated otherwise.

*Child-reported variable.

School principal reported variable.

"Objectively measured height and weight data.

***1 denotes reference category.

$\wedge$ Screen time was according to the American Association of Pediatrics guidelines. 
increasing PA and other health behaviours has emerged in the literature [37,38]. Hence, applying the socialecological theory, objective measures of PA, along with more robust environmental level factors should be considered for modelling PA.

This research used robust objectively measured data for calculating the child's weight status. While two large reviews $[7,20]$ have reported inconclusive or no relationship between weight status and PA levels, this research found that the weight status of the child was negatively associated with PA levels. Using objectively measured BMI data, being overweight or obese was associated with lower levels of PA. A possible explanation for this contrasting finding may be the use parent-reported height and weight data for children in other research, which has been found to lack validity and reliability when compared with objective anthropometric measures [39].

A key strength of this study is the large sample of nine year olds taken from the most comprehensive nationally representative children's health survey currently available in Ireland. According to the 2006 Census figures, there were 56,497 nine year old children resident in Ireland [23]. Thus, this data includes approximately one seventh of these children. Further, probability weights were applied to the data using survey data commands to ensure that the findings are national representative.

However, there are some limitations to this study. The data analysed for this research is cross-sectional, therefore, a causal relationship cannot be inferred. The sample only included nine year old children, hence, generalisability cannot be assumed for all children. Also, there was a relatively low response rate at the household level (57\%). The data has been weighted to overcome any issues arising from this; however, response bias may exist.

Further, the nature of the PA data collected does not correspond with WHO guidelines - 60 minutes of MVPA per day [10]. While self-report (by child) of the WHO PA levels was available, the data were not used for the dependent variable as it has been found that children under 10 are not reliable at recalling PA patterns, in particular PA intensity [40]. Validity studies have concluded that studies should use objective measures of PA, or if this is not feasible, rely on parental reports of child PA [41]. The PA data available for this research was primary caregiver reported as opposed to objectively measured data. The primary caregiver reported PA based on how many days in the last 14 the study child had achieved at least 20 minutes of hard physical activity. This self-report question was found to be reliable with acceptable validity when compared with accelerometer data [42]. Also, using this question, other Irish research has constructed PA categories in the same way [29]. Finally, this research provides a comprehensive list of individual level factors; however, some family and environmental level factors were not available such as the primary caregiver's PA patterns.

\section{Conclusions}

In conclusion, this study finds individual level factors; including many modifiable factors appear to have the strongest correlation with PA levels of nine year olds in Ireland. Remarkably, individual characteristics appear to predict PA levels when considered in the multiple domains. Future research should aim to use more robust objective measures to explore the usefulness of the interconnect that exists across these domains. In particular how the family and environmental settings could be useful facilitators for consistent individual level factors such as sports participation.

\section{Abbreviations}

BMI: Body mass index; PA: Physical activity; MVPA: Moderate-to-vigorous physical activity; TST: Total screen time; WHO: World Health Organisation.

\section{Competing interests}

The authors have indicated they have no conflicts of interest or financial relationships relevant to this article to disclose.

\section{Authors' contributions}

SC, EK and PK conceived the overall study design and SC analysed the data and drafted the initial manuscript. All authors have participated in the revising of the manuscript; and all have approved the manuscript as submitted.

\section{Acknowledgements}

The Growing Up in Ireland study is funded by the Government of Ireland through the Department of Children and Youth Affairs in association with the Department of Social Protection and the Central Statistics Office. We would like to thank the Health Research Board Centre for Health and Diet Research for funding this paper. We would also like to thank National Children's Research Centre, Crumlin, for facilitating international presentations of this research.

Received: 9 October 2013 Accepted: 28 March 2014

Published: 21 April 2014

\section{References}

1. Cavill N, Kahlmeier S, Racioppi F: Physical activity and health in Europe: evidence for action. WHO Regional Office for Europe: Copenhagen; 2006.

2. Brage S, Wedderkopp N, Ekelund U, Franks PW, Wareham NJ, Andersen LB, Froberg K: Features of the Metabolic Syndrome Are Associated With Objectively Measured Physical Activity and Fitness in Danish Children The European Youth Heart Study (EYHS). Diabetes care 2004, 27(9):2141-2148.

3. Telama R, Yang X, Viikari J, Valimaki I, Wanne O, Raitakari O: Physical activity from childhood to adulthood: a 21-year tracking study. Am J Prev Med 2005, 28(3):267-273.

4. Kelder SH, Perry CL, Klepp Kl, Lytle LL: Longitudinal tracking of adolescent smoking, physical activity, and food choice behaviors. Am J Public Health 1994, 84(7):1121-1126.

5. Dumith SC, Gigante DP, Domingues MR, Kohl HW: Physical activity change during adolescence: a systematic review and a pooled analysis. Int J Epidemiol 2011, 40(3):685-698.

6. Sullivan S: The physical activity of children: a study of 1,602 Irish schoolchildren aged 11-12 years. Ir Med J 2002, 95(3):78.

7. Sallis JF, Prochaska JJ, Taylor WC: A review of correlates of physical activity of children and adolescents. Med Sci Sports Exerc 2000, 32(5):963.

8. World Health Organization: Global Strategy on Diet, Physical Activity and Health. Recommended Amount of Physical Activity. Geneva: World Health Organization Press; 2010. 
9. World Health Organization: Global recommendations on physical activity for health. Geneva: World Health Organization Press; 2010.

10. National Physical Activity Guidelines Steering Group, Department of Health and Children, Health Services Executive: Get Ireland Active: National Guidelines on Physical Activity for Ireland. Dublin: The Stationery Office; 2009

11. Bauer KW, Neumark-Sztainer D, Fulkerson JA, Hannan PJ, Story M: Familial correlates of adolescent girls' physical activity, television use, dietary intake, weight, and body composition. Int J Behav Nutr Phys Act 2011, 8(1):25.

12. Department of Health: Health Survey for England 2008: Physical activity and fitness. London: The Stationery Office; 2010.

13. Riddoch CJ, Andersen LB, Wedderkopp N, Harro M, Klasson-Heggebo L, Sardinha LB, Cooper AR, Ekelund U: Physical activity levels and patterns of 9-and 15-yr-old European children. Med Sci Sports Exerc 2004, 36(1):86-92.

14. Woods CB, Tannehill D, Quinlan A, Moyna N, Walsh J: The Children's Sport Participation and Physical Activity Study (CSPPA). Research Report No. 1 Dublin: School of Health and Human Performance, Dublin City University and the Irish Sports Council; 2010:1

15. Van Sluijs EM, Skidmore PM, Mwanza K, Jones AP, Callaghan AM, Ekelund U, Harrison F, Harvey I, Panter J, Wareham NJ: Physical activity and dietary behaviour in a population-based sample of British 10-year old children: the SPEEDY study (Sport, Physical activity and Eating behaviour: environmental Determinants in Young people). BMC public health 2008, 8(1):388,

16. Troiano RP, Berrigan D, Dodd KW, Mâsse LC, Tilert T, McDowell M: Physical activity in the United States measured by accelerometer. Med Sci Sport Exerc 2008, 40(1):181.

17. Spence JC, Lee RE: Toward a comprehensive model of physical activity. Psychol Sport Exer 2003, 4(1):7-24

18. Bronfenbrenner U: Ecological models of human development. In International encyclopedia of education, Volume 3. 2nd edition. Oxford: Elsevier; 1994:37-43. Reprint in Gauvain, M. \& Cole, M (Eds.), Readings on the development of children, 2nd Ed, New York: Freeman.

19. Sallis JF, Owen N, Fisher EB: Ecological models of health behavior. Health Behav and Health Educ: Theory, research, and practice 2008, 4:465-486.

20. Van der Horst K, Paw M, Twisk JWR, Van Mechelen W: A brief review on correlates of physical activity and sedentariness in youth. Med Sci Sports Exerc 2007, 39(8):1241.

21. Sallis JF, Story M, Lou D: Study designs and analytic strategies for environmental and policy research on obesity, physical activity, and diet: recommendations from a meeting of experts. Am J Prev Med 2009, 36(2):S72-S77.

22. Williams J, Greene S, Doyle E, Harris E, Layte R, McCoy S, McCrory C, Murray A, Nixon E, O'Dowd T: Growing up in Ireland national longitudinal study of children. The Lives of 9-Year-Olds. Report 1. Dublin: The Stationery Office; 2009.

23. Irish Social Sciences Data Archive: Sample design and response in wave 1 of the nine year cohort of Growing Up in Ireland. 2010. Available at: http://www.ucd.ie/t4cms/ GUI-SampleDesign9YearCohort.pdf (accessed July 12 $2^{\text {th }}, 2013$ ).

24. Murray A, McCrory C, Thornton M, Williams J, Quail A, Swords L, Doyle E, Harris E: Growing Up in Ireland: Design, instrumentation and procedures for the child cohort. Dublin: Office of the Minister for Children and Youth Affairs Department of Health and Children; 2011.

25. Godin G, Shephard RJ: A simple method to assess exercise behavior in the community. Canadian journal of applied sport sciences Journal canadien des sciences appliquees au sport 1985, 10(3):141-146.

26. Godin G, Jobin J, Bouillon J: Assessment of leisure time exercise behavior by self-report: a concurrent validity study. Canadian journal of public health Revue canadienne de sante publique 1985, 77(5):359-362.

27. Sallis JF, Buono MJ, Roby JJ, Micale FG, Nelson JA: Seven-day recall and other physical activity self-reports in children and adolescents. Med Sci Sports Exerc 1993, 25(1):99.

28. Gordon-Larsen P, McMurray RG, Popkin BM: Determinants of adolescent physical activity and inactivity patterns. Pediatrics 2000, 105(6):e83-e83.

29. Lane A, Harrison M, Murphy N: Screen Time Increases Risk of Overweight and Obesity in Active and Inactive 9 Year Old Irish Children: A Cross Sectional Analysis. J Phys Activ Health 2013, in press.

30. Committee on Public Education: Children, Adolescents, and Television. Pediatrics 2001, 107(2):423-426.

31. Cole TJ, Bellizzi MC, Flegal KM, Dietz WH: Establishing a standard definition for child overweight and obesity worldwide: international survey. $B M J$ 2000, 320(7244):1240.
32. World Health Organization: Obesity: Preventing and Managing the Global Epidemic: Report of a WHO Consultation. Geneva, Switzerland: World Health Organization; 2000:894-898.

33. Biddle SJ, Whitehead S, O'Donovan TM, Nevill ME: Correlates of participation in physical activity for adolescent girls: A systematic review of recent literature. J Phys Act Health 2005, 2(4):421-432.

34. Kjønniksen L, Anderssen N, Wold B: Organized youth sport as a predictor of physical activity in adulthood. Scand J Med Sci Sports 2009, 19(5):646-654

35. Melkevik O, Torsheim T, lannotti RJ, Wold B: Is spending time in screenbased sedentary behaviors associated with less physical activity: a cross national investigation. Int J Behav Nutr Phys Act 2010, 7:46.

36. Vandewater EA, Shim M-S, Caplovitz AG: Linking obesity and activity level with children's television and video game use. J Adolesc 2004, 27(1):71-85

37. Gordon-Larsen P, Nelson MC, Page P, Popkin BM: Inequality in the built environment underlies key health disparities in physical activity and obesity. Pediatrics 2006, 117(2):417-424.

38. Sallis JF, Glanz K: The role of built environments in physical activity, eating, and obesity in childhood. Future Child 2006, 16(1):89-108.

39. Akinbami $L$, Ogden $C L$ : Childhood Overweight Prevalence in the United States: The Impact of Parent-reported Height and Weight. Obesity 2009, 17(8):1574-1580.

40. Telford A, Salmon J, Jolley D, Crawford D: Reliability and validity of physical activity questionnaires for children: the children's leisure activities study survey (CLASS). Pediatr Exerc Sci 2004, 16(1):64-78.

41. Loprinzi PD, Cardinal BJ: Measuring children's physical activity and sedentary behaviors. J Exerc SC Fit 2011, 9(1):15-23.

42. Prochaska JJ, Sallis JF, Long B: A physical activity screening measure for use with adolescents in primary care. Arch Pediatr Adolesc Med 2001, 155(5):554.

doi:10.1186/1471-2431-14-107

Cite this article as: Cadogan et al:: The effects of individual, family and environmental factors on physical activity levels in children: a crosssectional study. BMC Pediatrics 2014 14:107.

\section{Submit your next manuscript to BioMed Central and take full advantage of:}

- Convenient online submission

- Thorough peer review

- No space constraints or color figure charges

- Immediate publication on acceptance

- Inclusion in PubMed, CAS, Scopus and Google Scholar

- Research which is freely available for redistribution

Submit your manuscript at www.biomedcentral.com/submit
C Biomed Central 